\title{
Owning a Pet Is Associated with Changes in the Composition of Gut Microbiota and Could Influence the Risk of Metabolic Disorders in Humans
}

\author{
Javier Arenas-Montes ${ }^{1,2,3,4,+}+^{\mathbb{D}}$, Pablo Perez-Martinez ${ }^{1,2,3,4,+}+^{\mathbb{D}}$, Cristina Vals-Delgado ${ }^{1,2,3,4}$, \\ Juan Luis Romero-Cabrera 1,2,3,4 (D), Magdalena P. Cardelo 1,2,3,4 (D), Ana Leon-Acuña 1,2,3,4, \\ Gracia M. Quintana-Navarro 1,2,3,4 , Juan F. Alcala-Diaz 1,2,3,4 $\mathbb{1}$, Jose Lopez-Miranda 1,2,3,4 $\mathbb{1}$, \\ Antonio Camargo $1,2,3,4, *, \ddagger$ (D) and Francisco Perez-Jimenez $1,2,3,4, *, \ddagger(\mathbb{D}$
}

1 Lipids and Atherosclerosis Unit, Internal Medicine Unit, Reina Sofia University Hospital, 14004 Cordoba, Spain; ja.aremon@gmail.com (J.A.-M.); pablopermar@yahoo.es (P.P.-M.); cristina12bioq@gmail.com (C.V.-D.); juanluroca855@gmail.com (J.L.R.-C.); malenipc023@gmail.com (M.P.C.); analeoaa@hotmail.com (A.L.-A.); graciaquintana@hotmail.com (G.M.Q.-N.); jfalcala@gmail.com (J.F.A.-D.); md1lomij@uco.es (J.L.-M.)

2 Department of Medicine (Medicine, Dermatology and Otorhinolaryngology), University of Cordoba, 14071 Cordoba, Spain

check for

updates

Citation: Arenas-Montes, J.;

Perez-Martinez, P.; Vals-Delgado, C.; Romero-Cabrera, J.L.; Cardelo, M.P.;

Leon-Acuña, A.;

Quintana-Navarro, G.M.;

Alcala-Diaz, J.F.; Lopez-Miranda, J.;

Camargo, A.; et al. Owning a Pet Is

Associated with Changes in the

Composition of Gut Microbiota and

Could Influence the Risk of Metabolic Disorders in Humans. Animals 2021,

11, 2347. https://doi.org/10.3390/ ani11082347

Academic Editor:

Emanuela Prato-Previde

Received: 14 July 2021

Accepted: 6 August 2021

Published: 9 August 2021

Publisher's Note: MDPI stays neutral with regard to jurisdictional claims in published maps and institutional affiliations.

Copyright: (c) 2021 by the authors. Licensee MDPI, Basel, Switzerland. This article is an open access article distributed under the terms and conditions of the Creative Commons Attribution (CC BY) license (https:// creativecommons.org/licenses/by/ $4.0 /)$.
3 Maimonides Biomedical Research Institute of Cordoba (IMIBIC), 14004 Cordoba, Spain

4 CIBER Fisiopatologia de la Obesidad y Nutricion (CIBEROBN), Instituto de Salud Carlos III, 28029 Madrid, Spain

* Correspondence: antonio.camargo@imibic.org (A.C.); fperezjimenez@uco.es (F.P.-J.); Tel.: +34-957-213735 (A.C.); Fax: +34-957-012882 (F.P.-J)

+ These authors equally contributed to this work.

$\ddagger$ These authors equally contributed to this work.

Simple Summary: Metabolic syndrome represents a multicomponent disorder characterized by abdominal obesity, dyslipidemia, hypertension and impaired insulin sensitivity, which is associated with an increased risk of cardiovascular disease. The etiology of metabolic syndrome is the result of a complex interaction between genetic, metabolic and environmental factors. However, the relationship between the risk of suffering metabolic syndrome and owning pets has not been sufficiently studied, although being in contact with pets has been considered a protective factor against cardiovascular disease. Moreover, some evidence suggests that this protection might be due to favorable changes in the intestinal microbiota. Bearing this background in mind, in this work we hypothesized that people who live with pets harbor a different microbiota to those who do not own a pet, and this fact could reduce the risk of suffering metabolic syndrome.

Abstract: Pet ownership positively influences clinical outcomes in cardiovascular prevention. Additionally, cardiovascular disease (CVD) has been previously linked to microbiota dysbiosis. We evaluated the influence of owning a pet and its relationship with the intestinal microbiota. We analyzed the gut microbiota from 162 coronary patients from the CORDIOPREV study (NCT00924937) according to whether they owned pets $(n=83)$ or not $(n=79)$. The pet-owner group was further divided according to whether they owned dogs only $(n=28)$ or not $(n=55)$. A 7-item pet-owners test score was used. Patients who owned pets had less risk of metabolic syndrome (MetS) (OR = 0.462) and obesity $(\mathrm{OR}=0.519)$ and were younger $(p<0.001)$ than patients who did not own pets. Additionally, patients who owned dogs had less risk of MetS $(\mathrm{OR}=0.378)$ and obesity $(\mathrm{OR}=0.418)$ and were younger $(p<0.001)$ than patients who did not own pets. A preponderance of the genera Serratia and Coprococcus was found in the group of owners, while the genera Ruminococcus, an unknown genus of Enterobacteriaceae and Anaerotruncus were preponderant in the group of non-owners. In patients who owned dogs, Methanobrevibacter and two more genera, Coprococcus and Oscillospira, were more common. Our study suggests that the prevalence of MetS and obesity in CVD patients is lower in pet owners, and that pet ownership could be a protective factor against MetS through the shaping of the gut microbiota. Thus, owning a pet could be considered as a protective factor against cardiometabolic diseases. 
Keywords: gut microbiota; dysbiosis; pet; dog; cardiometabolic diseases

\section{Introduction}

The One Health concept has been established in recent years to highlight the interaction between people, animals and the environment, reaching with increasing repercussions into the field of public health. Thus, the World Small Animal Veterinary Association has proposed interdisciplinary collaborations to promote the health of people, animals and the environment, and the health benefits of people when interacting with companion animals, as well as translational research and comparative clinics in pets, seeking the benefits of animal and human (One) health as key objectives [1].

Cardiovascular disease (CVD) is currently a major world-wide epidemic, and is associated with type 2 diabetes mellitus (T2DM) and metabolic syndrome (MetS) [2]. Several epidemiological conditions, such as age, ethnicity, gender, diet, physical activity, amongst others, have been linked to MetS [3]. However, the relationship between the risk of suffering it and owning pets has not been sufficiently studied, although being in contact with pets has been considered a protective factor against CVD $[4,5]$ and other diseases in children, including allergies and obesity [6,7]. In fact, some evidence suggests that this protection might be due to favorable changes in the intestinal microbiota.

The gut microbiota is now recognized as an organ which is fully integrated in the metabolism of the host [8]. Recently, it has been proposed that dysbiosis may trigger the development of metabolic diseases such as obesity, MetS and T2DM [9-11]. Moreover, contact with pets, and particularly dogs, has been linked with changes in the gut microbiota composition, in relation to microbiota transfer and lifestyle habits [6,7]. In fact, it has even been proposed that pets and their owners share common intestinal bacteria [12].

Bearing this background in mind, we put forward the hypothesis that people who live with pets harbor a different microbiota to those who do not own a pet, and this fact could reduce the risk of suffering MetS and obesity. Thus, we aimed to evaluate the association between owning pets and the prevalence of MetS, and to explore if the microbiota composition was different between pet owners and those who do not own a pet. We therefore compared the composition of the human gut microbiota in both situations, in addition to the metabolic trait characteristics of metabolic syndrome, which are also related to cardiovascular disease.

\section{Methods}

\subsection{Study Participants}

We conducted this work within the framework of the CORDIOPREV study (Clinical Trials.gov.Identifier: NCT00924937), an ongoing prospective, randomized, open, controlled trial of 1002 patients receiving conventional treatment for coronary heart disease (CHD), who had their last coronary event over six months before enrolment in one of two different healthy dietary models (a Mediterranean (MED) diet and a low-fat (LF) diet) over a period of seven years.

The sample size was calculated following the method by Frieman et al. 1978 [13]. The proportion of the main variable studied (MetS) was stated as $58 \%$, as in previous results of CORDIOPREV [14]. Accepting an alpha risk of 0.05 and a beta risk of 0.1 in a two-sided test, 127 subjects were required in the observed group to recognize a difference greater than or equal to $15 \%$. We randomly selected a total list of 200 patients, on which we performed a 7-item questionnaire to evaluate pet ownership, from which a further 38 were discarded because no feces samples were available, or they had consumed antibiotics within 1 month preceding the sample collection. Therefore, we analyzed the baseline fecal samples of 162 patients (133 men and 29 women). The patients were divided into two groups, according to whether they owned a pet (pet-owner group) or not (non-pet-owner group): the former consisted of 83 patients (72 men and 11 women), while the latter was made up 
of 79 patients (61 men and 18 women). The metabolic characteristics of the subjects in the study are shown in Table 1. In addition, we divided the pet owners into one subgroup (dog owners), according to whether they owned dogs only (24 men and 4 women). 
Table 1. Baseline characteristics of the participants in the study. Values correspond to the mean \pm SD.

\begin{tabular}{|c|c|c|c|c|}
\hline- & Patients & Pets & No Pets & $p$-Value \\
\hline$n$ & 162 & 83 & 79 & $\mathrm{n} / \mathrm{a}$ \\
\hline Men/Women (n) & $133 / 29$ & $72 / 11$ & $61 / 18$ & 0.114 \\
\hline Diet (LF vs. MED) & $68 / 94$ & 35/48 (LF vs. MED) & 33/46 (LF vs. MED) & 0.959 \\
\hline T2DM (No vs. T2DM) & $49 / 113$ & 27/56 (No vs. T2DM) & 22/57 (No vs. T2DM) & 0.517 \\
\hline Metabolic syndrome (No vs. MetS) & $101 / 61$ & $59 / 24$ (No vs. MetS) & 42/37 (No vs. MetS) & 0.019 \\
\hline Obesity (No vs. Obesity) & $77 / 85$ & 46/37 (No vs. Obesity) & 31/48 (No vs. Obesity) & 0.039 \\
\hline Arterial hypertension (No vs. AHT) & $53 / 109$ & 33/50 (No vs. AHT) & 20/59 (No vs. AHT) & 0.050 \\
\hline Age (years) & $63.32 \pm 8.45$ & $60.86 \pm 8.21$ & $65.92 \pm 7.96$ & $<0.001$ \\
\hline Weight $(\mathrm{Kg})$ & $82.75 \pm 13.35$ & $83.15 \pm 14.2$ & $82.35 \pm 12.5$ & 0.708 \\
\hline$B M I\left(K g / m^{2}\right)$ & $30.36 \pm 3.94$ & $29.88 \pm 3.88$ & $30.85 \pm 3.96$ & 0.123 \\
\hline Serum triacylglycerols $(\mathrm{mg} / \mathrm{dL})$ & $129.32 \pm 88.49$ & $115.71 \pm 46.41$ & $143.44 \pm 115.89$ & 0.237 \\
\hline Total cholesterol $(\mathrm{mg} / \mathrm{dL})$ & $160.47 \pm 34.24$ & $156.87 \pm 31.73$ & $164.20 \pm 36.5$ & 0.191 \\
\hline HDL-cholesterol $(m g / d L)$ & $40.98 \pm 9.64$ & $41.29 \pm 9.83$ & $40.66 \pm 9.49$ & 0.745 \\
\hline LDL-cholesterol (mg/dL) & $93.63 \pm 27.92$ & $92.02 \pm 26.54$ & $95.37 \pm 29.42$ & 0.456 \\
\hline$C R P(m g / d L)$ & $2.77 \pm 3.81$ & $2.51 \pm 2.94$ & $3.03 \pm 4.54$ & 0.106 \\
\hline ISI & $4.07 \pm 2.62$ & $4.29 \pm 2.86$ & $3.80 \pm 2.29$ & 0.356 \\
\hline Systolic BP & $136.55 \pm 19.02$ & $134.00 \pm 17.58$ & $139.34 \pm 20.23$ & 0.084 \\
\hline Diastolic BP & $76.86 \pm 11.22$ & $77.11 \pm 9.89$ & $76.59 \pm 12.59$ & 0.776 \\
\hline
\end{tabular}

LF, low-fat diet; MED, Mediterranean diet; T2DM, type 2 diabetes mellitus; MetS, metabolic syndrome; AHT, arterial hypertension; BMI, body mass index; CRP, C-reactive protein; ISI, insulin sensitivity index and BP, blood pressure. The statistical differences between groups were evaluated by $\chi 2$ test (men/women) or one-way ANOVA.

\subsection{Diet Assessment}

Adherence to the Mediterranean diet (MED) was assessed by a validated 14-item questionnaire [15] and to the low-fat (LF) diet by a 9-point score. This was performed once before the start of the dietary intervention and then yearly. We used Spanish food composition tables and a validated food frequency questionnaire [16] to calculate the intake of fiber.

\subsection{Assessment of Pet Ownership}

We performed a 7-item questionnaire to evaluate pet ownership, including the kind and the number of pets, the length of time they had been living with their pets, and whether they kept the pets at home or outdoors. We evaluated the ownership of dogs, cats, birds and any other animals that owners considered as a pet (included in the questionary as "others"). In the case of patients who did not own a pet, we asked whether they had previously owned a pet, and, if so, the number of pets they had had, the number of years they had been living with them, and the time elapsed since they last lived with a pet (Table S1).

\subsection{Clinical Plasma Parameters}

To collect blood samples, we used tubes containing $0.1 \%$ EDTA, which were then centrifuged at $1500 \times g$ for $15 \mathrm{~min}$ at $4{ }^{\circ} \mathrm{C}$ to separate the plasma from the blood cells. From frozen samples, blinded to the team members, analytes were measured at the Lipid and Atherosclerosis Unit at Reina Sofia University Hospital by members of the laboratory research team, as previously described [17].

\subsection{DNA Extraction from Fecal Samples}

We gave the patients a box with carbonic ice and a sterile plastic bottle with a screw cap to collect fecal samples. This allowed us to keep the samples frozen after delivery to the laboratory staff and store them at $-80^{\circ} \mathrm{C}$. DNA was extracted using the QIAamp DNA Stool Mini Kit (Qiagen, Hilden, Germany), following the manufacturer's instructions. DNA samples were stored at $-20^{\circ} \mathrm{C}$, after quantification with the Nanodrop ND-1000 v3.5.2 spectrophotometer (Nanodrop Technology ${ }^{\circledR}$, Cambridge, UK), as previously described [17]. 


\subsection{Sequencing and Bioinformatics}

For each DNA (fecal) sample, we amplified by polymerase chain reaction the hypervariable regions $\mathrm{V} 3$ and $\mathrm{V} 4$ of the 16S rRNA gene using the primer pair 5'-TCGTCGGCAGCGTCAGATGTGTATAAGAGACAG-3' ${ }^{\prime}$ and $5^{\prime}$-GTCTCGTGGGCTCGG AGATGTGTATAAGAGACAG-3' [18], which was further sequenced on a MiSeq Illumina platform (Illumina, San Diego, CA, USA). Briefly, PCRs were performed using a KAPA HiFi HotStart ReadyMix (KAPABIOSYSTEMS), $1.25 \mu \mathrm{L}$ of extracted DNA (5 ng/ $\mu \mathrm{L}$ in $10 \mathrm{mM}$ Tris pH8.5) and $0.2 \mu \mathrm{M}$ of each primer, using the following cycle parameters: 3 minutes denaturation at $95^{\circ} \mathrm{C}$, followed by 25 cycles $\left(30 \mathrm{~s}\right.$ at $95^{\circ} \mathrm{C}, 30 \mathrm{~s}$ at $60^{\circ} \mathrm{C}, 30 \mathrm{~s}$ at $\left.72{ }^{\circ} \mathrm{C}\right)$ and a final extension at $72{ }^{\circ} \mathrm{C}$ for $5 \mathrm{~min}$. The $16 \mathrm{~S} \mathrm{~V} 3$ and V4 amplicon purification was performed using Agentcourt AMPure XP beads (Beckman Coulter). A second PCR attached dual indices and Illumina sequencing adapters using the Nextera XT Index Kit. This PCR was performed with a KAPA HiFi HotStart ReadyMix (KAPABIOSYSTEMS), with $5 \mu \mathrm{L}$ of the previous amplicon, $5 \mu \mathrm{L}$ of each Nextera XT Index Primer 1(N7xx) and $5 \mathrm{uL}$ of each Nextera XT Index Primer 2(S5xx), using the following cycle parameters: 3 min denaturation at $95^{\circ} \mathrm{C}$, followed by 8 cycles $\left(30 \mathrm{~s}\right.$ at $95^{\circ} \mathrm{C}, 30 \mathrm{~s}$ at $55^{\circ} \mathrm{C}, 30 \mathrm{~s}$ at $\left.72{ }^{\circ} \mathrm{C}\right)$, and a final extension at $72{ }^{\circ} \mathrm{C}$ for $5 \mathrm{~min}$, as previously described [19]. Sequence outputs were analyzed using the Quantitative Insights into Microbial Ecology (QIIME) program, version 1.9.1 [20], using QIIME default parameters. The $16 \mathrm{~S}$ paired reads were assembled using the script multiple_join_paired_ends.py, which joins forward and reverse demultiplexed reads. The output file was processed for quality filtering by split_libraries_fastq.py. High quality sequences were grouped into Operational Taxonomic Units (OTUs) with a sequence identity threshold of $97 \%$, and taxonomy was assigned by interrogating the high quality sequences with the Greengenes database (13_5) [21]. Bacterial richness and diversity across the samples were calculated using the Chao1, Simpson, and Shannon indexes [22]. Linear discriminant analysis (LDA) effect size (LEfSe) (http:/ / huttenhower.sph.harvard.edu/galaxy/ (accessed on 14 April 2020)) was used to compare groups at baseline and visualize the results using taxonomic bar charts and cladograms [23].

\subsection{Data Accesibility}

The sequences obtained in this study have been submitted to NCBI Sequence Read Archive (SRA) under the accession number PRJNA612957 (https://www.ncbi.nlm.nih. gov/sra/PRJNA612957).

\subsection{Statistical Analysis}

The PASW statistical software package, version 20.0 (IBM Inc., Chicago, IL, USA), was used for univariate statistical analyses of data. A $p$-value less than 0.05 was considered significant. The normal distribution of variables was assessed using the KolmogorovSmirnov test. Statistical differences in the main metabolic variables between the groups were evaluated using one-way ANOVA tests. All the quantitative data shown in this study were expressed as mean \pm standard deviation (SD). The Odds Ratio (OR), a measure of association that represents the odds that an outcome will occur given a particular exposure, compared to the odds of the outcome occurring in the absence of that exposure [24], was also obtained to calculate the risk of each group.

\section{Results}

\subsection{Baseline Characteristics of the Study Participants}

The differences in the main dietary, anthropometric and metabolic variables between groups are shown in Table 1. No statistically significant differences were observed in the main dietary and metabolic variables, but patients who owned a pet were younger than patients who did not $(p<0.001)$. In addition, patients who owned a pet had less risk of MetS $(\mathrm{OR}=0.241-\mathbf{0 . 4 6 2}-0.883 ; p=0.01)$ and obesity $(\mathrm{OR}=0.278-\mathbf{0 . 5 1 9}-0.971 ; p=0.03)$ than patients who did not. Moreover, differences between dog owners and non-pet owners are shown in Table 2. Dog owners were younger $(p<0.001)$ and had less risk of MetS 
$(\mathrm{OR}=0.144-\mathbf{0 . 3 7 8}-0.991 ; p=0.04)$ and obesity $(\mathrm{OR}=0.173-\mathbf{0 . 4 1 8}-\mathbf{1 . 0 1 0} ; p=0.0496)$ than patients who did not own a pet. The pets owned by participants were 56 dogs, 19 cats, 35 birds, and 11 pets classified as "others" (including foxes, turtles, fishes, etc.). Please note that a person could simultaneously own different species. Three pets had chronic diseases, three pets had any remarkable disease and four pets usually had digestive problems (diarrhea, constipation, vomiting).

Table 2. Differences between dog-owners and non-pet owners. Values correspond to the mean \pm SD.

\begin{tabular}{cccc}
\hline - & Dogs & No Pets & $p$-Value \\
\hline$n$ & 28 & 79 & $\mathrm{n} / \mathrm{a}$ \\
Men/Women $(n)$ & $24 / 4$ & $61 / 18$ & 0.339 \\
Diet (LF vs. MED) & $17 / 11$ (LF vs. MED) & $33 / 46$ (LF vs. MED) & 0.084 \\
T2DM (No vs. T2DM) & $8 / 20$ (No vs. T2DM) & $22 / 57$ (No vs. T2DM) & 0.942 \\
Metabolic syndrome (No vs. MetS) & $21 / 7$ (No vs. MetS) & $42 / 37$ (No vs. MetS) & $\mathbf{0 . 0 4 4}$ \\
Obesity (No vs. Obesity) & $17 / 11$ (No vs. Obesity) & $31 / 48$ (No vs. Obesity) & $\mathbf{0 . 0 4 9}$ \\
Arterial hypertension (No vs. AHT) & $10 / 18$ (No vs. AHT) & $20 / 59$ (No vs. AHT) & 0.293 \\
Age (years) & $60.17 \pm 7.70$ & $65.92 \pm 7.96$ & $<\mathbf{0 0 1}$ \\
Weight $($ Kg) & $81.85 \pm 12.16$ & $82.35 \pm 12.50$ & 0.860 \\
BMI $\left(\right.$ Kg/m $\left.^{2}\right)$ & $29.40 \pm 3.15$ & $30.85 \pm 3.96$ & 0.093 \\
Total cholesterol $(m g / d L)$ & $115.32 \pm 49.89$ & $143.44 \pm 115.89$ & 0.218 \\
HDL-cholesterol $(m g / d L)$ & $155.89 \pm 30.53$ & $164.20 \pm 36.50$ & 0.284 \\
LDL-cholesterol $(m g / d L)$ & $40.50 \pm 10.97$ & $40.66 \pm 9.49$ & 0.777 \\
CRP $(m g / d L)$ & $91.96 \pm 24.56$ & $95.37 \pm 29.42$ & 0.586 \\
ISI & $2.57 \pm 2.40$ & $3.03 \pm 4.54$ & 0.608 \\
Systolic BP & $4.03 \pm 2.13$ & $3.80 \pm 2.29$ & 0.715 \\
Diastolic BP & $134.11 \pm 17.48$ & $139.34 \pm 20.24$ & 0.232 \\
\end{tabular}

LF, low-fat diet; MED, Mediterranean diet; T2DM, type 2 diabetes mellitus; MetS, metabolic syndrome; AHT, arterial hypertension; BMI, body mass index; CRP, C-reactive protein; ISI, insulin sensitivity index and BP, blood pressure. The statistical differences between groups were evaluated by $\chi 2$ (men/women) test or one-way ANOVA.

\subsection{Microbiota Characteristics of the Study Participants}

In the pet-owners fecal microbiota samples, four phyla had relative sequence abundances greater than 1\%: Bacteroidetes (49.33\%), Firmicutes (43.16\%), Proteobacteria (4.85\%) and Verrucomicrobia (1.21\%). In the non-pet-owner gut microbiota samples, five phyla had relative abundances greater than 1\%: Bacteroidetes (48.54\%), Firmicutes (40.65\%), Proteobacteria (6.70\%), Verrucomicrobia (2.58\%), and Actinobacteria (1.11\%) (Figure 1). The fecal microbiota samples from pet owners revealed that the bacterial genera with a $>1 \%$ abundance were Bacteroides (24.28\%), Prevotella (11.60\%), an unknown genus of Ruminococcaceae $(9.08 \%)$, an unknown genus of Clostridiales (7.93\%), an unknown genus of Lachnospiraceae (3.74\%), Parabacteroides $(3.58 \%)$, Ruminococcus (3.02\%), Phascolarctobacterium (2.91\%), Faecalibacterium (2.78\%) and Lachnospira (2.78). By contrast, the intestinal microbiota samples of patients who did not own a pet showed that bacterial taxa with a $>1 \%$ abundance were Bacteroides (27.64\%), Prevotella $(8.52 \%)$, an unknown genus of Ruminococcaceae $(7.76 \%)$, an unknown genus of Clostridiales (6.37\%), Parabacteroides (4.10\%), an unknown genus of Lachnospiraceae (3.34\%), an unknown genus of Enterobacteriaceae (3.33\%) Lachnospira (2.92\%), Phascolarctobacterium (2.85\%), Ruminococcus (2.84\%) and Akkermansia (2.58\%). 


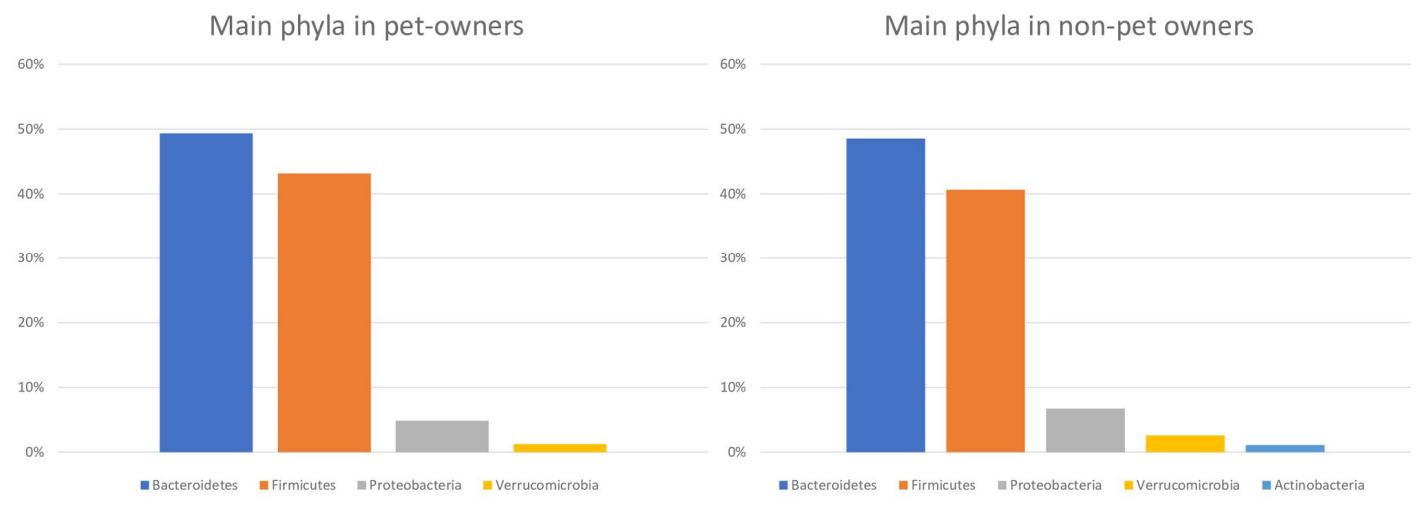

Figure 1. Each bar represents the mean relative sequence abundances of the phyla (only those phyla with an abundance higher than $1 \%$ are included).

\subsection{Differences in the Gut Microbiota between Pet Owners and Non-Pet Owners: LEfSe Analysis}

In order to evaluate changes in the human gut microbiota due to pet ownership, we assessed the global differences between patients who owned pets or did not. We used LEfSe to compare the estimated phylotypes between the groups and, as can be seen in Figure 2, a preponderance of Serratia and Coprococcus was significant in the owners while the non-owners had a preponderance of one genus of the Gammaproteobacteria class from the Enterobacteriaceae family, and two genera of the Clostridiales order, namely Ruminococcus and Anaerotruncus.
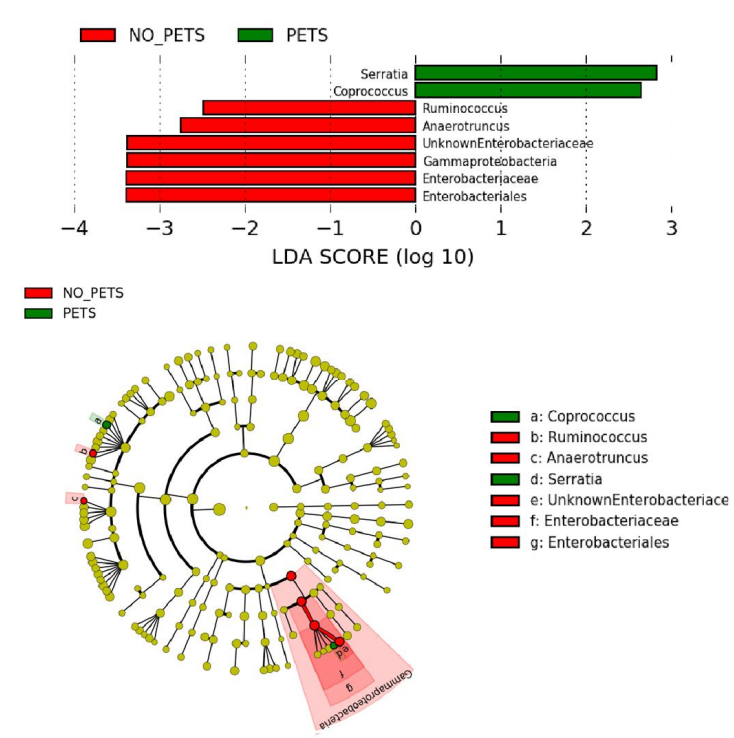

Figure 2. Cladogram representing the taxonomic hierarchical structure of the identified differences between pet owners and non-pet owners using Linear discriminant analysis effect size. Each filled circle represents one phylotype. Red, bacterial taxa statistically overrepresented in non-pet owners; green, bacterial taxa overrepresented in pet owners. Phylum and class are indicated in their names on the cladogram and the order, family, or genus are given in the key.

\subsection{Differences in the Gut Microbiota between Dog Owners and Non-Pet Owners: LEfSe Analysis}

To discern whether these differences in patients who owned pets were specific to dog owners, which were the majority group in the pet owners, we compared dog owners with non-pet owners (Figure 3). We used LEfSe to compare the estimated phylotypes of these groups. The dog owners' gut microbiota was characterized by a preponderance of the domain Archaea, one genus of the Methanobacteriales class, Methanobrevibacter, and two more genera, Coprococcus and Oscillospira, whereas the non-pet owners' intestinal microbiota was 
characterized by a preponderance of the domain Bacteria and an unknown genus of the Enterobacteriaceae family from the Gammaproteobacteria class.

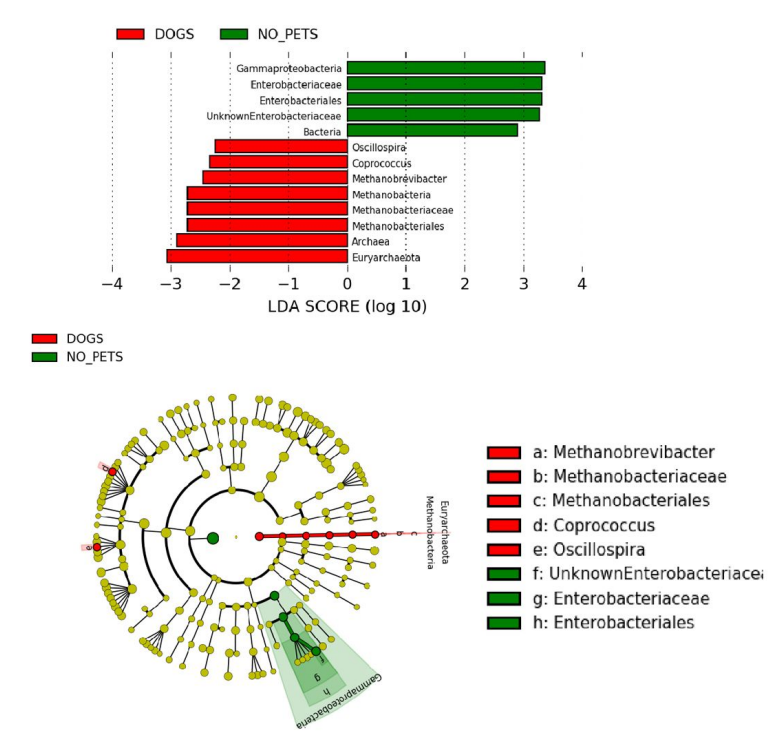

Figure 3. Cladogram representing the taxonomic hierarchical structure of the identified differences between dog owners and non-pet owners using Linear discriminant analysis effect size. Each filled circle represents one phylotype. Red, bacterial taxa statistically overrepresented in dog owners; green, bacterial taxa overrepresented in non-pet owners. Phylum and class are indicated in their names on the cladogram and the order, family, or genus are given in the key.

\section{Discussion}

This work provides evidence on the existence of specific differences in the intestinal microbiota composition according to whether the patient owns a pet or not, and its potential association with MetS. Moreover, we identified specific gut microbiota features associated particularly to owning a dog, and that pet ownership was linked to a lower risk of MetS and obesity.

Currently, the relationship between microbiota and disease is an emerging field in research. Studies in humans have identified a direct interaction between microbiota dysbiosis and the incidence of diseases such as nonalcoholic fatty liver disease and inflammatory bowel disease $[25,26]$. In addition, microbiota dysbiosis has also been related to a higher incidence of metabolic diseases and CVD, including MetS and obesity [27-29].

Previous data have indicated that factors, such as owning a pet, seem to affect the gut microbiota composition [30]. Additionally, it has also been described that pet ownership is associated with a lower risk of suffering CVD, mainly by providing social support and motivation for physical activity $[4,5]$. Despite this increasing knowledge, the potential improvement in the gut microbiota profile associated with pet ownership in cardiovascular disease patients has not yet been studied. In addition, to the best of our knowledge, none of the published studies have investigated the relationship between pet ownership and MetS.

It is interesting to note that differences in the gut microbiota in pet owners have been linked as a protective factor against the development of diseases such as allergies and obesity in infants [6,7]. In line with this, our results provide evidence that, in cardiovascular disease patients, pet owners and dog owners may have less risk of MetS than non-pet owners $(\mathrm{OR}=0.1-\mathbf{0 . 4 2}-0.94)$. Despite the difference in MetS prevalence between these groups, several MetS-related parameters, such as triacylglyceride levels and blood pressure were not statistically significant different, but were higher in patients with no pets; whereas, no differences were found in parameters such as HDL, presumably as a consequence of the differences in the HDL levels between sexes for this variable. In addition, although not statistically significantly different, others parameters such as LDL related to CVD, and CRP related to the inflammatory status, and in turn CVD, were higher in patients with no pets. 
Taken together, these observations suggest a better cardiometabolic status of pet owners and dog owners versus non-pet owners.

Furthermore, there was a small age difference between groups (5 years), which we did not consider relevant to influencing our findings. In addition, MetS and age were not statistically related in the study population in the comparison between the groups of pet owners and non-pet owners, and in the comparison between the groups of dog owners and non-pet owners.

Moreover, our study also showed that this difference in MetS prevalence between pet owners and the group with no pets was accompanied by differences in the gut microbiota. In fact, compared to the group of pet owners, the group with no pets was characterized by a gut microbiota with a preponderance of Ruminococcus, Anaerotruncus and an unknown genus of Enterobacteriaceae. The abundance of Ruminococcus and Anaerotruncus in the human microbiota has been previously related to a higher prevalence of MetS [19,31] and the abundance of Enterobacteriaceae has been positively linked to the development of obesity in children and pregnant women [32-34].

In our study, the gut microbiota of pet owners was characterized by higher levels of Coprococcus and Serratia. The genus Coprococcus has been shown to be a protective factor against MetS and T2DM [35] and is a short-chain fatty acid producer that modulates insulin resistance [36]. Although it is not a pathogenic genus, lower levels of Coprococcus are strongly associated with fasting serum levels of glycerol, monounsaturated fatty acids and saturated fatty acids, and inversely associated with polyunsaturated fatty acids [37]. Serratia, on the other hand, is a potentially pathogenic genus; however, its presence in healthy people has been related to it being a protective factor against obesity [38]. Thus, our findings are in line with previous studies describing an altered abundance of these bacterial taxa in metabolic disease, as the group of pet owners had less risk of MetS and obesity.

Considering the potential of different animals to change the human gut microbiota $[6,7,30]$, and particularly the fact that they have been shown to be a protective factor against various diseases such as allergies [39], we explored the specific bacterial differences between the subgroup of patients who only owned dogs, versus the non-pet owners in general. Here, our data showed that the genera Oscillospira, Coprococcus and Methanobrevibacter, together with the Archaea domain, were found in higher proportions in the gut microbiota of dog owners. The higher prevalence of Archaea is probably due to the higher proportion of Methanobrevibacter, an interesting methanogen genus and SCFA producer [40] which has been linked to a lower body mass index (BMI) and lower levels of triglycerides, while a lower fecal concentration of this genus has been found in prediabetic subjects [37].

In addition, the Oscillospira genus has been correlated with leanness, lower BMI and a lower prevalence of obesity, and is able to degrade host glycans (such as fucose, sialic acids and glucuronic acid) [41,42].

In agreement with these findings, the increased presence of Oscillospira, Methanobrevibacter and Coprococcus in the samples of dog owners could explain the lower prevalence of MetS and obesity in this population [35-37]. These genera are not related to human infections. Very few studies have explored the gut microbiota of people who come into contact with pets, and in all of them, infants were studied. However, these studies have shown that two of the four genera that our data showed as more prevalent in pet owners and dog owners, Coprococcus and Oscillospira, have been previously linked to pet ownership, which reinforces the hypothesis of the transfer of microbiota from pets to owners. In fact, higher levels of Coprococcus and Oscillospira have been previously linked to infants who live in a household where there is a dog [6,7].

By contrast, the other two genera, Serratia and Methanobrevibacter, have not yet been reported as being higher in humans who have contact with pets. Even though Serratia has not been identified in humans in contact with pets, this genus has been found in healthy dogs, which constitute the majority of the pets owned by our patients, which suggests that it could be transferred from pets to their owners $[43,44]$. Like Serratia, a higher abundance 
of Methanobrevibacter has not been found previously in humans living with dogs; however, this genus can also be present in the dog microbiota [45].

Diet consumption is an important factor that can modulate the intestinal microbiota. Specifically, a LF diet has been related to an increased proportion of the Prevotella genus and F. prausnitzii genera and a decreased proportion of the Roseburia genus, whereas the MED diet has been related to decreased levels of the Prevotella genus and increased levels of P. distasonis, Roseburia and Oscillospira genera [46]. However, from these, only Oscillospira has been related to pet ownership [7], and no other differences in these genera were found between groups, presumably because of the fact that there were not statistical differences in diet consumption between the groups of pet owners.

Our study has limitations and could be considered a preliminary study. One limit lies in the fact that the population in which we performed the study has CVD and may already present several potential alterations in their gut microbiota associated with this disease. Further studies are needed to clarify the relationship between cardiovascular disease and pet ownership, with particular reference to changes in the gut microbiota that may be produced as a consequence. Moreover, the study was performed in a reduced sample size population, which limits our findings. In addition, our study did not consider the age of the animals, which should also be considered in future studies. Thus, it will be necessary to replicate the study in other populations and undertake validation in a cohort without cardiovascular disease and closer to the general population.

\section{Conclusions}

Our study suggests that the prevalence of MetS and obesity in CVD patients is lower in pet owners, and that pet ownership could be a protective factor against MetS by shaping the gut microbiota. Moreover, the microbiota profile found in pet owners and dog owners was consistent with the prevalence of obesity and MetS in this population. Thus, owning a pet may be considered as a protective factor against cardiometabolic diseases.

Supplementary Materials: The following are available online at https:/ /www.mdpi.com/article/10 .3390/ani11082347/s1, Table S1: A 7-item questionnaire to evaluate pet ownership, including the kind and the number of pets, the length of time they had been living with their pets, and whether they kept the pets at home or outdoors.

Author Contributions: Conceptualization, P.P.-M., A.C. and F.P.-J.; methodology, J.A.-M., P.P.-M. and A.C.; software, G.M.Q.-N., J.F.A.-D., J.L.-M., and A.C.; validation, J.L.-M. and F.P.-J.; formal analysis, G.M.Q.-N., J.F.A.-D., J.L.-M., and A.C.; investigation, J.A.-M., C.V.-D., J.L.R.-C., M.P.C., and A.L.-A.; resources; J.L.-M. and F.P.-J.; data curation, J.A.-M., C.V.-D., J.L.R.-C., M.P.C., and A.L.-A.; writing — original draft preparation, J.A.-M., P.P.-M., and A.C.; writing—-review and editing, J.L.-M. and F.P.-J.; visualization, J.A.-M., P.P.-M., J.F.A.-D., J.L.-M., A.C., and F.P.-J.; supervision; J.L.-M. and F.P.-J.; project administration, P.P.-M., A.C. and F.P.-J.; funding acquisition, A.C., J.L.-M., P.P.-M. and F.P.-J. All authors have read and agreed to the published version of the manuscript.

Funding: The CORDIOPREV study is supported by the Fundación Patrimonio Comunal Olivarero, Junta de Andalucía (Consejería de Salud, Consejería de Agricultura y Pesca, Consejería de Innovación, Ciencia y Empresa), Diputaciones de Jaén y Córdoba, Centro de Excelencia en Investigación sobre Aceite de Oliva y Salud and Ministerio de Medio Ambiente, Medio Rural y Marino, Gobierno de España; Instituto de Salud Carlos III (CP14/00114, CPII19/00007, PI19/00299 and DTS19/00007 to A.C.; PI13/00619 to F P-J; PI16/01777 to F.P.-J. and P.P.-M.). Ministerio de Economía y Competitividad (AGL2012/39615, PIE14/00005, and PIE14/00031 to J.L.-M.; AGL2015-67896-P to J.L.-M. and A.C.); Consejería de Innovación, Ciencia y Empresa, Proyectos de Investigación de Excelencia, Junta de Andalucía (CVI-7450 to J.L.-M.); and by the Fondo Europeo de Desarrollo Regional (FEDER). Antonio Camargo is supported by an ISCIII research contract (Programa Miguel-Servet CP14/00114 and CPII19/00007). We would like to thank the Córdoba branch of the Biobank of the Sistema Sanitario Público de Andalucía (Andalusia, Spain) for providing the biological human samples.

Institutional Review Board Statement: The eligibility criteria, design and methods of the CORDIOPREV clinical trial have been reported elsewhere (13). All the patients gave their informed consent in 
writing to participate in the study. The trial protocol and all the amendments were approved by the local ethics committees, following the Helsinki Declaration and good clinical practice.

Informed Consent Statement: Informed consent was obtained from all subjects involved in the study.

Data Availability Statement: The sequences obtained in this study have been submitted to NCBI Sequence Read Archive (SRA) under the accession number PRJNA612957 (https:/ / www.ncbi.nlm. nih.gov/sra/PRJNA612957).

Acknowledgments: The CIBEROBN is an initiative of the Instituto de Salud Carlos III, Madrid, Spain. We would like to thank the Córdoba branch of the Biobank of the Sistema Sanitario Público de Andalucía (Andalusia, Spain) for providing the biological human samples. We would also like to thank the EASP (Escuela Andaluza de Salud Pública), Granada, Spain.

Conflicts of Interest: The authors declare that they have no competing interests.

\section{References}

1. $\quad$ Bartges, J.; Kushner, R.F.; Michel, K.E.; Sallis, R.; Day, M.J. One Health Solutions to Obesity in People and Their Pets. J. Comp. Pathol. 2017, 156, 326-333. [CrossRef]

2. Grundy, S.M.; Cleeman, J.I.; Daniels, S.R.; Donato, K.A.; Eckel, R.H.; Franklin, B.A.; Gordon, D.J.; Krauss, R.M.; Savage, P.J.; Smith, S.C., Jr.; et al. Diagnosis and management of the metabolic syndrome: An American Heart Association/National Heart, Lung, and Blood Institute Scientific Statement. Circulation 2005, 112, 2735-2752. [CrossRef]

3. Perez-Martinez, P.; Mikhailidis, D.P.; Athyros, V.G.; Bullo, M.; Couture, P.; Covas, M.I.; de Koning, L.; Delgado-Lista, J.; DiazLopez, A.; Drevon, C.A.; et al. Lifestyle recommendations for the prevention and management of metabolic syndrome: An international panel recommendation. Nutr. Rev. 2017, 75, 307-326. [CrossRef]

4. Yeh, T.L.; Lei, W.T.; Liu, S.J.; Chien, K.L. A modest protective association between pet ownership and cardiovascular diseases: A systematic review and meta-analysis. PLoS ONE 2019, 14, e0216231. [CrossRef] [PubMed]

5. Levine, G.N.; Allen, K.; Braun, L.T.; Christian, H.E.; Friedmann, E.; Taubert, K.A.; Thomas, S.A.; Wells, D.L.; Lange, R.A. Pet ownership and cardiovascular risk: A scientific statement from the American Heart Association. Circulation 2013, 127, $2353-2363$. [CrossRef]

6. Azad, M.B.; Konya, T.; Maughan, H.; Guttman, D.S.; Field, C.J.; Sears, M.R.; Becker, A.B.; Scott, J.A.; Kozyrskyj, A.L. Infant gut microbiota and the hygiene hypothesis of allergic disease: Impact of household pets and siblings on microbiota composition and diversity. Allergy Asthma Clin. Immunol. 2013, 9, 15. [CrossRef] [PubMed]

7. Tun, H.M.; Konya, T.; Takaro, T.K.; Brook, J.R.; Chari, R.; Field, C.J.; Guttman, D.S.; Becker, A.B.; Mandhane, P.J.; Turvey, S.E.; et al. Exposure to household furry pets influences the gut microbiota of infant at 3-4 months following various birth scenarios. Microbiome 2017, 5, 40. [CrossRef] [PubMed]

8. Tremaroli, V.; Bäckhed, F. Functional interactions between the gut microbiota and host metabolism. Nature 2012, 489, 242-249. [CrossRef]

9. Bajzer, M.; Seeley, R.J. Physiology: Obesity and gut flora. Nature 2006, 444, 1009-1010. [CrossRef] [PubMed]

10. Caesar, R.; Fåk, F.; Bäckhed, F. Effects of gut microbiota on obesity and atherosclerosis via modulation of inflammation and lipid metabolism. J. Intern. Med. 2010, 268, 320-328. [CrossRef] [PubMed]

11. Diamant, M.; Blaak, E.E.; de Vos, W.M. Do nutrient-gut-microbiota interactions play a role in human obesity, insulin resistance and type 2 diabetes? Obes. Rev. 2011, 12, 272-281. [CrossRef]

12. Caugant, D.A.; Levin, B.R.; Selander, R.K. Distribution of multilocus genotypes of Escherichia coli within and between host families. J. Hyg. 1984, 92, 377-384. [CrossRef] [PubMed]

13. Freiman, J.A.; Chalmers, T.C.; Smith, H.; Kuebler, R.R. The importance of beta, the type II error and sample size in the design and interpretation of the randomized control trial. Survey of 71 "negative" trials. N. Engl. J. Med. 1978, 299, 690-694. [CrossRef] [PubMed]

14. Delgado-Lista, J.; Perez-Martinez, P.; Garcia-Rios, A.; Alcala-Diaz, J.F.; Perez-Caballero, A.I.; Gomez-Delgado, F.; Fuentes, F.; Quintana-Navarro, G.; Lopez-Segura, F.; Ortiz-Morales, A.M.; et al. CORonary Diet Intervention with Olive oil and cardiovascular PREVention study (the CORDIOPREV study): Rationale, methods, and baseline characteristics: A clinical trial comparing the efficacy of a Mediterranean diet rich in olive oil versus a low-fat diet on cardiovascular disease in coronary patients. Am. Heart J. 2016, 177, 42-50. [CrossRef]

15. Schröder, H.; Fitó, M.; Estruch, R.; Martínez-González, M.A.; Corella, D.; Salas-Salvadó, J.; Lamuela-Raventós, R.; Ros, E.; Salaverría, I.; Fiol, M.; et al. A short screener is valid for assessing Mediterranean diet adherence among older Spanish men and women. J. Nutr. 2011, 141, 1140-1145. [CrossRef]

16. Fernández-Ballart, J.D.; Piñol, J.L.; Zazpe, I.; Corella, D.; Carrasco, P.; Toledo, E.; Perez-Bauer, M.; Martínez-González, M.A.; Salas-Salvadó, J.; Martín-Moreno, J.M. Relative validity of a semi-quantitative food-frequency questionnaire in an elderly Mediterranean population of Spain. Br. J. Nutr. 2010, 103, 1808-1816. [CrossRef] 
17. Haro, C.; Rangel-Zúñiga, O.A.; Alcalá-Díaz, J.F.; Gómez-Delgado, F.; Pérez-Martínez, P.; Delgado-Lista, J.; Quintana-Navarro, G.M.; Landa, B.B.; Navas-Cortés, J.A.; Tena-Sempere, M.; et al. Intestinal Microbiota Is Influenced by Gender and Body Mass Index. PLoS ONE 2016, 11, e0154090. [CrossRef]

18. Klindworth, A.; Pruesse, E.; Schweer, T.; Peplies, J.; Quast, C.; Horn, M.; Glöckner, F.O. Evaluation of general 16S ribosomal RNA gene PCR primers for classical and next-generation sequencing-based diversity studies. Nucleic Acids Res. 2013, 41, e1. [CrossRef]

19. Santos-Marcos, J.A.; Haro, C.; Vega-Rojas, A.; Alcala-Diaz, J.F.; Molina-Abril, H.; Leon-Acuña, A.; Lopez-Moreno, J.; Landa, B.B.; Tena-Sempere, M.; Perez-Martinez, P.; et al. Sex Differences in the Gut Microbiota as Potential Determinants of Gender Predisposition to Disease. Mol. Nutr. Food Res. 2019, 63, e1800870. [CrossRef]

20. Caporaso, J.G.; Kuczynski, J.; Stombaugh, J.; Bittinger, K.; Bushman, F.D.; Costello, E.K.; Fierer, N.; Peña, A.G.; Goodrich, J.K.; Gordon, J.I.; et al. QIIME allows analysis of high-throughput community sequencing data. Nat. Methods 2010, 7, 335-336. [CrossRef]

21. McDonald, D.; Price, M.N.; Goodrich, J.; Nawrocki, E.P.; DeSantis, T.Z.; Probst, A.; Andersen, G.L.; Knight, R.; Hugenholtz, P. An improved Greengenes taxonomy with explicit ranks for ecological and evolutionary analyses of bacteria and archaea. ISME J. 2012, 6, 610-618. [CrossRef]

22. Hammer, Ø.; Harper, D.A.T.; Ryan, P.D. PAST: Paleontological Statistics Software Package for Education and Data Analysis. Palaeontol. Electron. 2001, 4, 9.

23. Segata, N.; Izard, J.; Waldron, L.; Gevers, D.; Miropolsky, L.; Garrett, W.S.; Huttenhower, C. Metagenomic biomarker discovery and explanation. Genome Biol. 2011, 12, R60. [CrossRef]

24. Szumilas, M. Explaining odds ratios. J. Can. Acad. Child. Adolesc. Psychiatry 2010, 19, 227-229.

25. Zuo, T.; Ng, S.C. The Gut Microbiota in the Pathogenesis and Therapeutics of Inflammatory Bowel Disease. Front. Microbiol. 2018, 9, 2247. [CrossRef] [PubMed]

26. Alisi, A.; Ceccarelli, S.; Panera, N.; Nobili, V. Causative role of gut microbiota in non-alcoholic fatty liver disease pathogenesis. Front. Cell. Infect. Microbiol. 2012, 2, 132. [CrossRef]

27. Ley, R.E.; Turnbaugh, P.J.; Klein, S.; Gordon, J.I. Microbial ecology: Human gut microbes associated with obesity. Nature 2006, 444, 1022-1023. [CrossRef]

28. Festi, D.; Schiumerini, R.; Eusebi, L.H.; Marasco, G.; Taddia, M.; Colecchia, A. Gut microbiota and metabolic syndrome. World J. Gastroenterol. 2014, 20, 16079-16094. [CrossRef]

29. Koren, O.; Spor, A.; Felin, J.; Fåk, F.; Stombaugh, J.; Tremaroli, V.; Behre, C.J.; Knight, R.; Fagerberg, B.; Ley, R.E.; et al. Human oral, gut, and plaque microbiota in patients with atherosclerosis. Proc. Natl. Acad. Sci. USA 2011, 108, 4592-4598. [CrossRef]

30. Oh, C.; Lee, K.; Cheong, Y.; Lee, S.-W.; Park, S.-Y.; Song, C.-S.; Choi, I.-S.; Lee, J.-B. Comparison of the Oral Microbiomes of Canines and Their Owners Using Next-Generation Sequencing. PLoS ONE 2015, 10, e0131468. [CrossRef]

31. Zupancic, M.L.; Cantarel, B.L.; Liu, Z.; Drabek, E.F.; Ryan, K.A.; Cirimotich, S.; Jones, C.; Knight, R.; Walters, W.A.; Knights, D.; et al. Analysis of the gut microbiota in the old order Amish and its relation to the metabolic syndrome. PLoS ONE 2012, 7, e43052. [CrossRef]

32. Kalliomäki, M.; Collado, M.C.; Salminen, S.; Isolauri, E. Early differences in fecal microbiota composition in children may predict overweight. Am. J. Clin. Nutr. 2008, 87, 534-538. [CrossRef]

33. Karlsson, C.L.; Onnerfält, J.; Xu, J.; Molin, G.; Ahrné, S.; Thorngren-Jerneck, K. The microbiota of the gut in preschool children with normal and excessive body weight. Obesity 2012, 20, 2257-2261. [CrossRef]

34. Santacruz, A.; Collado, M.C.; García-Valdés, L.; Segura, M.T.; Martín-Lagos, J.A.; Anjos, T.; Martí-Romero, M.; Lopez, R.M.; Florido, J.; Campoy, C.; et al. Gut microbiota composition is associated with body weight, weight gain and biochemical parameters in pregnant women. Br. J. Nutr. 2010, 104, 83-92. [CrossRef]

35. Fiorucci, S.; Distrutti, E. Bile Acid-Activated Receptors, Intestinal Microbiota, and the Treatment of Metabolic Disorders. Trends Mol. Med. 2015, 21, 702-714. [CrossRef] [PubMed]

36. Lippert, K.; Kedenko, L.; Antonielli, L.; Kedenko, I.; Gemeier, C.; Leitner, M.; Kautzky-Willer, A.; Paulweber, B.; Hackl, E. Gut microbiota dysbiosis associated with glucose metabolism disorders and the metabolic syndrome in older adults. Benef. Microbes 2017, 8, 545-556. [CrossRef]

37. Org, E.; Blum, Y.; Kasela, S.; Mehrabian, M.; Kuusisto, J.; Kangas, A.J.; Soininen, P.; Wang, Z.; Ala-Korpela, M.; Hazen, S.L.; et al. Relationships between gut microbiota, plasma metabolites, and metabolic syndrome traits in the METSIM cohort. Genome Biol. 2017, 18, 70. [CrossRef] [PubMed]

38. Chiu, C.M.; Huang, W.C.; Weng, S.L.; Tseng, H.C.; Liang, C.; Wang, W.C.; Yang, T.; Yang, T.L.; Weng, C.T.; Chang, T.H.; et al. Systematic analysis of the association between gut flora and obesity through high-throughput sequencing and bioinformatics approaches. Biomed. Res. Int. 2014, 2014, 906168. [CrossRef]

39. Ownby, D.R.; Johnson, C.C.; Peterson, E.L. Exposure to dogs and cats in the first year of life and risk of allergic sensitization at 6 to 7 years of age. JAMA 2002, 288, 963-972. [CrossRef] [PubMed]

40. Goodrich, J.K.; Waters, J.L.; Poole, A.C.; Sutter, J.L.; Koren, O.; Blekhman, R.; Beaumont, M.; Van Treuren, W.; Knight, R.; Bell, J.T.; et al. Human genetics shape the gut microbiome. Cell 2014, 159, 789-799. [CrossRef]

41. Kohl, K.D.; Amaya, J.; Passement, C.A.; Dearing, M.D.; McCue, M.D. Unique and shared responses of the gut microbiota to prolonged fasting: A comparative study across five classes of vertebrate hosts. FEMS Microbiol. Ecol. 2014, 90, 883-894. [CrossRef] 
42. Konikoff, T.; Gophna, U. Oscillospira: A Central, Enigmatic Component of the Human Gut Microbiota. Trends Microbiol. 2016, 24, 523-524. [CrossRef]

43. Handl, S.; German, A.J.; Holden, S.L.; Dowd, S.E.; Steiner, J.M.; Heilmann, R.M.; Grant, R.W.; Swanson, K.S.; Suchodolski, J.S. Faecal microbiota in lean and obese dogs. FEMS Microbiol. Ecol. 2013, 84, 332-343. [CrossRef]

44. Abramson, A.L.; Isenberg, H.D.; McDermott, L.M. Microbiology of the canine nasal cavities. Rhinology 1980, 18, 143-150. [CrossRef]

45. Schmidt, M.; Unterer, S.; Suchodolski, J.S.; Honneffer, J.B.; Guard, B.C.; Lidbury, J.A.; Steiner, J.M.; Fritz, J.; Kölle, P. The fecal microbiome and metabolome differs between dogs fed Bones and Raw Food (BARF) diets and dogs fed commercial diets. PLoS ONE 2018, 13, e0201279. [CrossRef]

46. Santos-Marcos, J.A.; Perez-Jimenez, F.; Camargo, A. The role of diet and intestinal microbiota in the development of metabolic syndrome. J. Nutr. Biochem. 2019, 70, 1-27. [CrossRef] 